\title{
RNA-directed DNA Methylation
}

\author{
Huiming Zhang ${ }^{1}$ and Jian-Kang Zhu, ${ }^{1}$, \\ ${ }^{1}$ Department of Botany and Plant Sciences, Institute for Integrative Genome Biology, University of \\ California, Riverside, CA 92521, USA
}

\section{Abstract}

DNA methylation is an important epigenetic mechanism for silencing transposons and other repetitive elements, and for stable repression of specific transgenes and endogenous genes. Plants can utilize small interfering RNAs (siRNAs) to guide de novo DNA methyltransferases for the establishment of sequence-specific DNA methylation. Genetic and biochemical approaches have identified many components involved in RNA-directed DNA methylation (RdDM). These components function in one or more of the following three aspects: biogenesis of siRNAs, production of scaffold RNAs, and the assembly of an effector complex that involves the complementary pairing between the guide siRNAs and nascent scaffold RNAs and which recruits the DNA methyltransferases. Recent studies not only unveiled new molecular players and novel interactions, but also suggested spatial and temporal segregation of the RdDM process within the nucleus.

\section{Introduction}

DNA encodes genetic information that needs to be selectively transcribed during different developmental stages and in response to various environmental stimuli. Epigenetic silencing through formation of heterochromatin, which involves DNA methylation and/or repressive histone modifications, plays an important role in suppressing unfavorable transcription of genes and preventing the proliferation of transposable elements. In plants, 24-nt small interfering RNAs (siRNAs) are known to guide DNA methyltransferases to the siRNAgenerating genomic loci and other loci that are homologous to the siRNAs for de novo DNA methylation, a pathway named RNA-directed DNA methylation (RdDM) [1-4]. Since the first report of RdDM phenomenon in 1994 [1], research to date has unveiled major steps in this albeit still incomplete pathway. Paradoxically, transcription is a prominent theme for transcriptional silencing through the RdDM pathway. This transcription involves three different DNA-dependent RNA polymerases that produce not only siRNA precursors but also scaffold RNAs that are necessary for RdDM. In this review, we describe the current understanding of RdDM and focus on recent discoveries that advanced the mechanistic insights into this pathway.

\section{Overview of RdDM Pathway}

In plants and mammals, DNA methylation is commonly found on transposons and other repetitive DNA elements [5, 6]. In plants, DOMAIN REARRANGED

\footnotetext{
(C) 2011 Elsevier Ltd. All rights reserved.

*corresponding author: Zhu, Jian-Kang (jian-kang.zhu@ucr.edu).
}

Publisher's Disclaimer: This is a PDF file of an unedited manuscript that has been accepted for publication. As a service to our customers we are providing this early version of the manuscript. The manuscript will undergo copyediting, typesetting, and review of the resulting proof before it is published in its final citable form. Please note that during the production process errors may be discovered which could affect the content, and all legal disclaimers that apply to the journal pertain. 
METHYLTRANSFERASE 2 (DRM2), an ortholog of the mammalian de novo DNA methyltransferase DNMT3, is primarily responsible for catalyzing de novo DNA methylation [7]. DNA Methyltransferase 1 (MET1), which is an ortholog of mammalian DNMT1, and the plant-specific CMT3, are CG- and CHG-specific maintenance methyltransferases, respectively. However, MET1 and CMT3 appear to also play a role in de novo methylation [8-10]. Recruitment of DRM2 and possibly other methyltransferases to their target sites can be mediated by the RdDM pathway. Because CHH methylation cannot be sustained by the maintenance methyltransferases, it must occur de novo every time there is DNA replication, and guidance by siRNAs ensures preservation of specific $\mathrm{CHH}$ methylation patterns in daughter cells. RdDM pathway has recently been shown to mediate faithful restoration of DNA methylation defects caused by mutations in the chromatin remodeler gene $D D M 1$ [11]. In Arabidopsis, many molecular components required for RdDM have been identified, resulting in a delineated pathway (Fig. 1). In general, the pathway consists of three phases: biogenesis of siRNAs, scaffold RNA production, and recognition of homologous ARGONAUTE 4 (AGO4)- or AGO6-bound siRNAs by the nascent scaffold RNA which leads to formation of a guiding complex that recruits DRM2 to the target loci (Fig. 1).

\section{Biogenesis of siRNAs}

The 24-nt siRNAs require RNA-DEPENDENT RNA POLYMERASE 2 (RDR2) and DICER-LIKE 3 (DCL3) for accumulation [12] . While most of them require only RNA polymerase IV (Pol IV), some also require Pol V, Pol II and other components of the effector complex [13-18]. Pol IV and Pol V have unique subunits but also share several subunits between them and with Pol II [19-21]. The plant-specific Pol IV and Pol V clearly evolved from the more ubiquitous Pol II. In non-plant eukaryotes, where Pol IV and Pol V are not present but small RNAs are produced and can also direct DNA methylation and/or heterochromatic histone modifications [22-24], Pol II alone likely serves all of the roles of the three or even four plant polymerases (Pol II, Pol IV, Pol V and RDR2).

Current understanding favors a role for Pol IV in initiating siRNA biogenesis by generating an abberant, single-stranded RNA (ssRNA) [3, 4, 13]. Although mutations in Pol IV drastically reduce 24-nt siRNA abundance [14, 15, 25], Pol IV transcripts have yet to be identified. Templates for Pol IV transcription remain unclear, with methylated DNA, nascent ssRNA, or double-stranded RNA (dsRNA) being candidates [4, 13]. Upon generation of ssRNA by Pol IV, RDR2 is thought to convert the ssRNA into doublestranded RNA (dsRNA), which is then processed into 24-nucleotide (nt) siRNAs by DCL3 [3,4]. HUA ENHANCER 1 (HEN1) is then involved in the pathway through 3'-terminal ribose methylation that stabilizes the siRNAs [26]. Alternative to Pol IV and RDR2 mediation, dsRNAs can also be derived from RNA polymerase II (Pol II) transcription of inverted repeats $[13,15]$. In addition to these canonical siRNA-generating components, genetic screening recently identified RNA-DIRECTED DNA METHYLATION 4 (RDM4; also known as DMS4) as a regulatory factor for RNA polymerases II and V [16, 17]. RDM4 encodes a conserved putative transcription factor, whose loss-of-function not only impairs the RdDM pathway due to effects on Pol V transcription of intergenic sequences but also causes pleiotropic developmental defects in Arabidopsis due to effects on Pol II transcription of protein-coding genes. Early steps in siRNA biogenesis also involves CLASSY 1 (CLSY1, also known as CHR38), a putative SNF2-like chromatin remodeling factor, which has been suggested to function together with Pol IV or RDR2 [18]. 


\section{Scaffold RNA Production}

Scaffold RNAs originated from intergenic non-coding sequences appear to be required for silencing adjacent siRNA-generating loci [27-29]. Pol V and Pol II both have been shown to generate scaffold RNAs for RdDM [27, 29]. NRPE1 (NUCLEAR RNA POLYMERASE E1), the largest subunit of Pol V, is necessary for scaffold RNA generation at several loci. In addition, NRPE1 was shown to associate with the DNA templates and scaffold RNAs. These results suggest that the nascent scaffold RNAs are Pol V transcripts [27]. Pol II appears to generate scaffold RNAs at intergenic low-copy-number repeat loci [28]. In a weak mutant allele of NRPB2 (the second largest subunit of Pol II), occupancy of Pol IV at heterochromatic loci where siRNA production depends on Pol IV but not Pol V was reduced compared to that in wild type Arabidopsis; similarly, occupancy of Pol V at regions adjacent to these siRNA-generating loci was reduced in the $n r p b 2$ mutant [28]. The study suggested that the different RNA polymerases cooperate during the RdDM process and the scaffold transcripts generated by Pol II may help recruit Pol IV and Pol V to the RdDM target loci. RdDM at low-copy-number repeat loci thus involves at least two types of scaffold RNAs, one produced by Pol II and the other produced by Pol V. Pol V-dependent synthesis of scaffold RNAs requires the putative chromatin-remodeling protein DRD1 (DEFECTIVE IN RNA-DIRECTED DNA METHYLATION 1), the chromosome hinge domain protein DMS3 (DEFECTIVE MERISTEM SILENCING 3), and RDM1 [27, 28, 30-33]; whereas Pol II requires DRD1 but not DMS3 for scaffold RNA production [28]. The effect of RDM1 on Pol II-dependent scaffold RNAs has not been examined.

Biogenesis of siRNAs is not essential for scaffold RNA production, as demonstrated in Arabidopsis mutants defective in DCL3, RDR2, or the largest subunit of Pol IV NRPD1 (NUCLEAR RNA POLYMERASE D 1) [27]. Scaffold RNAs in yeast not only dock siRNA binding but also function as precursors of siRNAs [34, 35]. Theoretically Pol V or Pol II transcripts may provide templates for Pol IV transcription or directly act as siRNA precursors by serving as RDR2 templates. Alternatively, the scaffold transcripts could affect siRNA accumulation by recruiting Pol IV, or by influencing DNA methylation or heterochromatic histone modification marks that are important for siRNA biogenesis. Consistent with these possibilities, siRNA accumulation from low-copy-number repeats and intergenic sequences is reduced in nrpel and nrpb2 mutants of Arabidopsis.

\section{The Guiding Complex and DRM2 Recruitment}

siRNAs loaded onto AGO4 or AGO6 are thought to serve as sequence-specific guides during RdDM by pairing with complementary DNA targets or nascent scaffold RNAs from the DNA targets $(1-4,36)$. Recent evidence favors the RNA-RNA pairing model $[5,8,27$, 28]. In parallel to the base-pairing between siRNAs and nascent scaffold RNAs, specific protein interactions are required for establishing the guiding complex that recruits DRM2. Among members of such a complex are AGO4 and AGO6, and proteins involved in synthesizing or binding scaffold RNAs, including Pol V, Pol II, DRD1, DMS3, RDM1 and KOW-DOMAIN CONTAINING TRANSCRIPTION FACTOR 1 (KTF1). The siRNAscaffold RNA interaction is further strengthened by two other interactions. First, AGO4 binds strongly to NRPE1 and KTF1, both of which contain many GW/WG repeats, the socalled "Ago hook" domains [34, 38, 39]. Several GW/WG motifs are also present in NRPB2, which may help recruit AGO4 to Pol II-generated scaffold transcripts [28]. Second, the extensive GW/WG repeats-containing tails of NRPE1 and KTF1 can also bind scaffold RNAs [38]. Recent studies indentified IDN2 (also known as RDM12) as another putative effector protein in RdDM. IDN2 contains an XS domain known to bind dsRNAs with 5'overhangs and is therefore suggested to facilitate siRNA-scaffold RNA base-pairing [31, 
40]. Alternatively, IDN2 may facilitate the function of RDR2, a role analogous to the XS domain-containing protein SGS3 in facilitating the function of RDR6 [40].

Dysfunction of RDM1 reduces abundance of "Pol IV- and Pol V-dependent" type I siRNAs, but does not affect "Pol IV-dependent but Pol V-independent" type II siRNAs [41], suggesting that RDM1, like Pol V and AGO4, may influence reinforcement, rather than initiation, of siRNAs production and is therefore an effector protein for RdDM. Consistently, RDM1 interacts with NRPB1 of Pol II [41], and also co-purifies with DRD1 and DMS3, forming a complex necessary for Pol V transcription [33]. Importantly, RDM1 coimmunoprecipitates and co-localizes with AGO4 as well as DRM2 in the nucleus [41], suggesting that RDM1 is part of a larger complex that contains DRM2. The observation that RDM1 binds single-stranded methylated DNA in vitro [41] also supports a central role of RDM1 in the silencing complex. Pol II and/or Pol V transcription necessitates a transient unwinding of DNA to generate single-stranded DNA, to which RDM1 may bind in vivo. Alternatively, RDM1 may bind single-stranded DNA during DNA replication, which would imply a coupling between RdDM and DNA replication.

\section{Spatial and Temporal Distribution of RdDM Foci}

Cytological analyses have recently suggested spatial and temporal patterns of RdDM foci. Co-localization of siRNAs with RDR2, DCl3, AGO4, and NRPE1 was observed in perinucleolar bodies, which are proposed as siRNA processing centers [42]. DRM2 co-localizes with AGO4, NRPE1, and NRPD/E2 (the second largest subunit common to Pol IV and Pol $\mathrm{V})$ in peri-nucleolar bodies [43, 44], indicating active RdDM in these structures. Consistently, co-localization of RDM1 with DRM2, AGO4, and NRPE1 was also observed in the peri-nucleolar bodies [41]. These peri-nucleolar bodies, which have also been referred to as the AGO4-NRPD1b or AB bodies [43], are distinct from Cajal bodies where AGO4 also localizes, since NRPE1 does not co-localize with a marker protein that is characteristic of Cajal bodies [43, 44]. Interestingly, NRPB1 co-localizes with RDM1 and AGO4 in discrete foci in the nucleoplasm but not the peri-nucleolar bodies [41]. DRM2 is also found in these nucleoplasmic foci [41]. These observations suggest that there is active RdDM in the nucleoplasmic foci and Pol II may be an important part of this. In contrast, neither NRPE1 nor NRPD1 has a strong co-localization with AGO4 or RDM1 in the nucleoplasm. This suggests that RdDM in the nucleoplasmic foci is associated with Pol II but not Pol IV or Pol V transcription. It is possible that some RdDM target loci are methylated in the perinucleolar bodies, while other RdDM target loci are methylated in the nucleoplasmic foci. The RdDM in peri-nucleolar bodies would depend on scaffold RNAs produced by Pol V whereas Pol II produced scaffold RNAs would function in RdDM in the nucleoplasmic foci. Alternatively, the activities in the peri-nucleolar bodies and nucleoplasmic foci may be sequential. That is, part of the RdDM process may take place in the peri-nucleolar bodies and then the target sequences may exit to the nucleoplasm for further modification. Recent evidence suggests that while highly repetitive sequences may be silenced by Pol V-mediated RdDM in peri-nucleolar bodies, RdDM silencing of intergenic low-copy-number loci appear to also depend on Pol II in the nucleoplasm [27, 28, 41] (Fig. 2).

RdDM also appears to have a temporal pattern. NRPE4, a subunit required for Pol V, enters peri-nucleolar bodies only under certain circumstances, as indicated by the presence of NRPE4 immunofluorescence signal foci, which overlap with the peri-nucleolar bodies containing AGO4 and NRPE1, in 50\% of the nuclei examined [45]. Such a pattern suggests that Pol V-dependent RdDM is needed only at certain cell stages or in certain cell types. Although the latter can be time-independent, developmental regulation of heterochromatin de-compaction was observed in Arabidopsis [46, 47, 48]. For example, the leaf chromocenter reduces its compaction during floral transition [47]. Similarly, 5S rDNA 
de-condenses on the first day post-germination and subsequently requires NRPD/E2a, a subunit common to Pol IV and Pol V, for recondensation [48].

\section{Conclusions}

While recent studies improved our knowledge of RdDM, new questions arose whereas preexisting questions remain. For instance, how is siRNA biogenesis initiated? What features define whether or not a locus may generate 24-nt siRNAs and whether or not it may be transcribed by Pol II and/or Pol V to produce scaffold RNAs? Do Pol II or Pol V transcripts provide templates for Pol IV or RDR2 in generating siRNAs? What specific proteins in the guiding complex interact with DRM2 to recruit it to RdDM target sites? There are likely additional components in the RdDM pathway, which remain to be identified through further genetic screens and biochemical studies. Distinct peri-nucleolar and nucleoplasmic foci are associated with RdDM. What is the relationship between the peri-nucleolar and nucleoplasmic sites and their RdDM activities? There may also be dynamic changes in RdDM components and activities during the cell cycle. Further cell biological studies are necessary for a better understanding of the spatial and temporal segregation of RdDM components and activities.

\section{Acknowledgments}

We apologize to colleagues whose work we were unable to include because of space limitations. The work in J-K Z lab was supported by National Institutes of Health grants R01GM070795 and R01GM059138.

\section{References and recommended reading}

Papers of particular interest, published within the past two years, have been highlighted as:

* of special interest

** of outstanding interest

1. Wassenegger M, Heimes S, Riedel L, Sänger HL. RNA-directed de novo methylation of genomic sequences in plants. Cell. 1994; 76:567-576. [PubMed: 8313476]

2. Chinnusamy V, Zhu JK. RNA-directed DNA methylation and demethylation in plants. Sci China C Life Sci. 2009; 52:331-343. [PubMed: 19381459]

3. Law JA, Jacobsen SE. Establishing, maintaining and modifying DNA methylation patterns in plants and animals. Nat Rev Genet. 2010; 11:204-220. [PubMed: 20142834]

4. Matzke M, Kanno T, Huettel B, Daxinger L, Matzke AJ. Targets of RNA-directed DNA methylation. Curr Opin Plant Biol. 2007; 10:512-519. [PubMed: 17702644]

5. Zhang X, et al. Genome-wide high-resolution mapping and functional analysis of DNA methylation in Arabidopsis. Cell. 2006; 126:1189-1201. [PubMed: 16949657]

6. Suzuki MM, Bird A. DNA methylation landscapes: provocative insights from epigenomics. Nat Rev Genet. 2008; 9:465-476. [PubMed: 18463664]

7. Cao X, Jacobsen SE. Role of the Arabidopsis DRM methyltransferases in de novo DNA methylation and gene silencing. Curr Biol. 2002; 12:1138-1144. [PubMed: 12121623]

8. Zhu JK. Epigenome sequencing comes of age. Cell. 2008; 133:395-397. [PubMed: 18455978]

9. Cao X, Aufsatz W, Zilberman D, Mette MF, Huang MS, Matzke M, Jacobsen SE. Role of the DRM and CMT3 methyltransferases in RNA-directed DNA methylation. Curr Biol. 2003; 13:2212-2217. [PubMed: 14680640]

10. Aufsatz W, Mette MF, Matzke AJ, Matzke M. The role of MET1 in RNA-directed de novo and maintenance methylation of CG dinucleotides. Plant Mol. Biol. 2004; 54:793-804. [PubMed: 15604652] 
11. Teixeira FK, et al. A role for RNAi in the selective correction of DNA methylation defects. Science. 2009; 323:1600-1604. [PubMed: 19179494]

12. Xie Z, Johansen LK, Gustafson AM, Kasschau KD, Lellis AD, Zilberman D, Jacobsen SE, Carrington JC. Genetic and functional diversification of small RNA pathways in plants. PLoS Biol. 2004; 2:E104. [PubMed: 15024409]

13. Pikaard CS, Haag JR, Ream T, Wierzbicki AT. Roles of RNA polymerase IV in gene silencing. Trends Plant Sci. 2008; 13:390-397. [PubMed: 18514566]

14*. Mosher RA, Schwach F, Studholme D, Baulcombe DC. PolIVb influences RNA-directed DNA methylation independently of its role in siRNA biogenesis. Proc Natl Acad Sci U S A. 2008; 105:3145-3150. [PubMed: 18287047] [Deep sequencing of siRNAs in Arabidopsis revealed a role of Pol V in reinforcing Pol IV-dependent siRNA production. Pol V was also shown to be necessary for generation of siRNAs in ca. 1/3 of the $>4000$ identified loci.]

15. Zhang X, Henderson IR, Lu C, Green PJ, Jacobsen SE. Role of RNA polymerase IV in plant small RNA metabolism. Proc Natl Acad Sci U S A. 2007; 104:4536-4541. [PubMed: 17360559]

16*. He XJ, Hsu YF, Zhu S, Liu HL, Pontes O, Zhu J, Cui X, Wang CS, Zhu JK. A conserved transcriptional regulator is required for RNA-directed DNA methylation and plant development. Genes Dev. 2009; 23:2717-2722. [PubMed: 19903758] [Together with Ref. [14*], this study identified a conserved transcription factor that affects both RdDM and plant development through regulating Pol V and Pol II transcription.]

17*. Kanno T, et al. RNA-directed DNA methylation and plant development require an IWR1-type transcription factor. EMBO Rep. 2010; 11:65-71. [PubMed: 20010803]

18. Smith LM, Pontes O, Searle I, Yelina N, Yousafzai FK, Herr AJ, Pikaard CS, Baulcombe DC. An SNF2 protein associated with nuclear RNA silencing and the spread of a silencing signal between cells in Arabidopsis. Plant Cell. 2007; 19:1507-1521. [PubMed: 17526749]

19**. Ream TS, Haag JR, Wierzbicki AT, Nicora CD, Norbeck AD, Zhu JK, Hagen G, Guilfoyle TJ, Pasa-Tolić L, Pikaard CS. Subunit compositions of the RNA-silencing enzymes Pol IV and Pol V reveal their origins as specialized forms of RNA polymerase II. Mol Cell. 2009; 33:192-203. [PubMed: 19110459] [This study identified the shared as well as unique subunits among the plant-specific Pol IV, Pol V, and the conserved Pol II.]

20. Lahmy S, Bies-Etheve N, Lagrange T. Plant-specific multisubunit RNA polymerase in gene silencing. Epigenetics. 2010; 5:4-8. [PubMed: 20173420]

21*. Huang L, Jones AM, Searle I, Patel K, Vogler H, Hubner NC, Baulcombe DC. An atypical RNA polymerase involved in RNA silencing shares small subunits with RNA polymerase II. Nat Struct Mol Biol. 2009; 16:91-93. [PubMed: 19079263] [Pol V shares some but not all subunits with Pol II. Mass spectrometric study also identified novel Pol V-associated proteins.]

22. Moazed D. Small RNAs in transcriptional gene silencing and genome defence. Nature. 2009; 457:413-420. [PubMed: 19158787]

23. Grewal SI, Elgin SC. Transcription and RNA interference in the formation of heterochromatin. Nature. 2007; 447:399-406. [PubMed: 17522672]

24. Aravin AA, Hannon GJ. Small RNA silencing pathways in germ and stem cells. Cold Spring Harb Symp Quant Biol. 2008; 73:283-290. [PubMed: 19270082]

$25 *$. Haag JR, Pontes O, Pikaard CS. Metal A and metal B sites of nuclear RNA polymerases Pol IV and Pol V are required for siRNA-dependent DNA methylation and gene silencing. PLoS One. 2009; 4:e4110. [PubMed: 19119310] [Site-directed mutagenesis and in vivo functional assays demonstrated two active sites in Pol IV and Pol V, suggesting that active RNA polymerase activities are required for Pol IV and Pol $V$ to function in RdDM.]

26. Yang Z, Ebright YW, Yu B, Chen X. HEN1 recognizes 21-24 nt small RNA duplexes and deposits a methyl group onto the 2' $\mathrm{OH}$ of the 3' terminal nucleotide. Nucleic Acids Res. 2006; 34:667-675. [PubMed: 16449203]

27**. Wierzbicki AT, Haag JR, Pikaard CS. Noncoding transcription by RNA polymerase Pol IVb/Pol V mediates transcriptional silencing of overlapping and adjacent genes. Cell. 2008; 135:635-648. [PubMed: 19013275] [Identified Pol V transcripts for the first time. This study suggested a critical role of nascent Pol V transcripts as scaffold RNAs in RdDM pathway.] 
$28 * *$. Zheng B, Wang Z, Li S, Yu B, Liu JY, Chen X. Intergenic transcription by RNA polymerase II coordinates Pol IV and Pol V in siRNA-directed transcriptional gene silencing in Arabidopsis. Genes Dev. 2009; 23:2850-2860. [PubMed: 19948763] [This study established an important role of Pol II in regulating RdDM through generation of scaffold RNAs. Together with Ref. [16**], this study suggested critical roles of DNA-dependent RNA polymerases in RdDM by generating scaffold RNAs.]

29*. Wierzbicki AT, Ream TS, Haag JR, Pikaard CS. RNA polymerase V transcription guides ARGONAUTE4 to chromatin. Nat Genet. 2009; 41:630-634. [PubMed: 19377477] [The siRNA carrier AGO4 was shown to be recruited to silencing target loci through a process that is dependent on Pol V transcripts.]

30. Kanno T, Mette MF, Kreil DP, Aufsatz W, Matzke M, Matzke AJ. Involvement of putative SNF2 chromatin remodeling protein DRD1 in RNA-directed DNA methylation. Curr Biol. 2004; 14:801-805. [PubMed: 15120073]

31. Ausin I, Mockler TC, Chory J, Jacobsen SE. IDN1 and IDN2 are required for de novo DNA methylation in Arabidopsis thaliana. Nat Struct Mol Biol. 2009; 16:1325-1327. [PubMed: 19915591]

32. Kanno T, Bucher E, Daxinger L, Huettel B, Böhmdorfer G, Gregor W, Kreil DP, Matzke M, Matzke AJ. A structural-maintenance-of-chromosomes hinge domain-containing protein is required for RNA-directed DNA methylation. Nat Genet. 2008; 40:670-675. [PubMed: 18425128]

33*. Law JA, Ausin I, Johnson LM, Vashisht AA, Zhu JK, Wohlschlegel JA, Jacobsen SE. A protein complex required for polymerase $\mathrm{V}$ transcripts and RNA- directed DNA methylation in Arabidopsis. Curr Biol. 2010; 20:951-956. [PubMed: 20409711] [RDM1 was shown to form a protein complex with DRD1 and DMS3, and the complex is required for Pol V transcription.]

34. Bühler M, Verdel A, Moazed D. Tethering RITS to a nascent transcript initiates RNAi- and heterochromatin-dependent gene silencing. Cell. 2006; 125:873-886. [PubMed: 16751098]

35. Bühler M, Haas W, Gygi SP, Moazed D. RNAi-dependent and -independent RNA turnover mechanisms contribute to heterochromatic gene silencing. Cell. 2007; 129:707-721. [PubMed: 17512405]

36. Zheng X, Zhu J, Kapoor A, Zhu JK. Role of Arabidopsis AGO6 in siRNA accumulation, DNA methylation and transcriptional gene silencing. EMBO J. 2007; 26:1691-1701. [PubMed: 17332757]

37. El-Shami M, Pontier D, Lahmy S, Braun L, Picart C, Vega D, Hakimi MA, Jacobsen SE, Cooke R, Lagrange T. Reiterated WG/GW motifs form functionally and evolutionarily conserved ARGONAUTE-binding platforms in RNAi-related components. Genes Dev. 2007; 21:2539-2544. [PubMed: 17938239]

38*. He XJ, Hsu YF, Zhu S, Wierzbicki AT, Pontes O, Pikaard CS, Liu HL, Wang CS, Jin H, Zhu JK. An effector of RNA-directed DNA methylation in arabidopsis is an ARGONAUTE 4- and RNAbinding protein. Cell. 2009; 137:498-508. [PubMed: 19410546] [Genetic and biochemical analyses showed that KTF1, transcriptional elongation factor-like protein, functions as an effector protein in RdDM through interaction with AGO4 as well as binding to scaffold RNAs.]

39. Bies-Etheve N, Pontier D, Lahmy S, Picart C, Vega D, Cooke R, Lagrange T. RNA-directed DNA methylation requires an AGO4-interacting member of the SPT5 elongation factor family. EMBO Rep. 2009; 10:649-654. [PubMed: 19343051]

40. Zheng Z, Xing Y, He XJ, Li W, Hu Y, Yadav SK, Oh J, Zhu JK. An SGS3-like protein functions in RNA-directed DNA methylation and transcriptional gene silencing in Arabidopsis. Plant J. 2010; 62:92-99. [PubMed: 20059743]

41**. Gao Z, et al. An RNA polymerase II- and AGO4-associated protein acts in RNA-directed DNA methylation. Nature. 2010; 465:106-109. [PubMed: 20410883] [This study identified a putative single-stranded methyl DNA-binding protein, RDM1 that associates with DRM2, AGO4 and Pol II, providing a physical link between DRM2 and the rest of the silencing complex. In addition, differential co-localization of RDM1 with other key RdDM components suggested a spatial segregation of the RdDM process between the peri-nucleolar bodies and nucleoplasmic foci.]

42. Pontes O, Li CF, Nunes PC, Haag J, Ream T, Vitins A, Jacobsen SE, Pikaard CS. The Arabidopsis chromatin-modifying nuclear siRNA pathway involves a nucleolar RNA processing center. Cell. 2006; 126:79-92. [PubMed: 16839878] 
43**. Li CF, Henderson IR, Song L, Fedoroff N, Lagrange T, Jacobsen SE. Dynamic regulation of ARGONAUTE4 within multiple nuclear bodies in Arabidopsis thaliana. PLoS Genet. 2008; 4:e27. [PubMed: 18266474] [Co-localization of AGO4 with NRPE1, NRPE2, and DRM2 was observed, leading to the identification of peri-nucleolar RdDM foci. These peri-nucleolar foci are different from another type of AGO4-containing structure, Cajal bodies, since NRPE1 does not co-localize with the small nuclear RNA binding protein U2" that is characteristic to Cajal bodies.]

44. Li CF, Pontes O, El-Shami M, Henderson IR, Bernatavichute YV, Chan SW, Lagrange T, Pikaard CS, Jacobsen SE. An ARGONAUTE4-containing nuclear processing center colocalized with Cajal bodies in Arabidopsis thaliana. Cell. 2006; 126:93-106. [PubMed: 16839879]

45*. He XJ, Hsu YF, Pontes O, Zhu J, Lu J, Bressan RA, Pikaard C, Wang CS, Zhu JK. NRPD4, a protein related to the RPB4 subunit of RNA polymerase II, is a component of RNA polymerases IV and V and is required for RNA-directed DNA methylation. Genes Dev. 2009; 23:318-330. [PubMed: 19204117] [Enrichment of NRPD/E4 in the peri-nucleolar RdDM foci was only observed in about $50 \%$ of nuclei examined, suggesting that RdDM at peri-nucleolar foci is not uniform in all cell stages/types, thus supporting a possible temporal pattern of RdDM.]

46. Tessadori F, Chupeau MC, Chupeau Y, Knip M, Germann S, van Driel R, Fransz P, Gaudin V. Large-scale dissociation and sequential reassembly of pericentric heterochromatin in dedifferentiated Arabidopsis cells. J Cell Sci. 2007; 120:1200-1208. [PubMed: 17376962]

47. Tessadori F, Schulkes RK, van Driel R, Fransz P. Light-regulated large-scale reorganization of chromatin during the floral transition in Arabidopsis. Plant J. 2007; 50:848-857. [PubMed: 17470059]

48. Douet J, Blanchard B, Cuvillier C, Tourmente S. Interplay of RNA Pol IV and ROS1 during postembryonic 5S rDNA chromatin remodeling. Plant Cell Physiol. 2008; 49:1783-1791. [PubMed: 18845569]

49. Pontes O, Costa-Nunes P, Vithayathil P, Pikaard CS. RNA polymerase V functions in Arabidopsis interphase heterochromatin organization independently of the 24-nt siRNA-directed DNA methylation pathway. Mol Plant. 2009; 2:700-710. [PubMed: 19825650]

50. Tsai MC, Manor O, Wan Y, Mosammaparast N, Wang JK, Lan F, Shi Y, Segal E, Chang HY. Long noncoding RNA as modular scaffold of histone modification complexes. Science. 2010; 329:689-693. [PubMed: 20616235] 


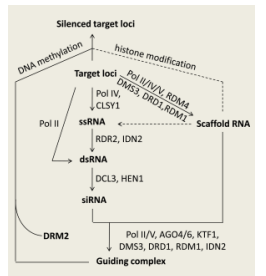

Figure 1.

A schematic diagram of the RdDM pathway. RdDM target loci are transcribed for biogenesis of 24-nt siRNAs and scaffold RNAs. Subsequently, siRNAs bind to the homologous nascent scaffold RNAs, resulting in a guiding complex that recruits DRM2 for de novo DNA methylation. Scaffold RNAs may also cause repressive histone modifications that also contribute to the formation of heterochromatin $[49,50]$. Proteins involved in each process are as indicated. Scaffold RNAs may also be copied to generate siRNAs. 


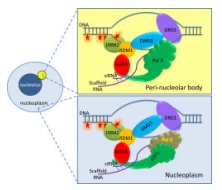

Figure 2.

Spatial segregation of RdDM. Pol V, but not Pol II, is associated with RdDM in perinucleolar bodies (yellow circle in Left Panel). In the nucleoplasm, Pol II and KTF1 are associated with RdDM. The RdDM effector complex is diagrammed to function at the transcription bubble created by Pol II or Pol V. Red stars represent DNA methylation. The GW/WG-rich tails of NRPE1 of Pol V and KTF1 are shown to bind AGO4 and scaffold RNAs. 